\title{
Full-field ERG in diabetic retinopathy: a screening tool?
}

\author{
Radouil Tzekov ${ }^{1}$
}

Received: 21 April 2015 / Accepted: 22 April 2015 /Published online: 9 May 2015

(C) Springer-Verlag Berlin Heidelberg 2015

Diabetes mellitus and its complications remains one of the major public health priorities of the modern era. Diabetic retinopathy (DR) is a frequent and major sight-threatening complication with increasing prevalence. In the US, projections of the National Eye Institute indicate that from 2010-2050, the number of Americans with DR is expected to nearly double, from 7.7 million to 14.6 million [1]. An important aspect of increased DR prevalence is that, in most cases, DR is subjectively asymptomatic until it has caused profound and often irreversible damage and visual loss. Therefore, development of objective methods to detect changes in retinal function and correlating them with the severity and progression of DR is important, providing a potentially beneficial approach either as an add-on to existing screening or as a guide in therapeutic decisions during the course of the disease.

Full-field electroretinography (ERG) is still the most common clinical visual electrodiagnostic test worldwide, and it has been used extensively as an objective method to assess visual function in the past, including in DR [2]. Thus, any advancement in our understanding of how ERG correlates with other DR clinical signs and symptoms would be helpful in refining and improving the clinical management of DR. The article "Photopic full-field electroretinography and optical coherence tomography" by Jansson et al. [3] is definitely an attempt in that regard and, considering the number of patients involved, is the first major full-field ERG study in DR to appear in the peer-reviewed literature during the past 10 years. The author's decision to conduct such a study and compare photopic ERG responses with high-

Radouil Tzekov

rtzekov@health.usf.edu

1 Department of Ophthalmology, University of South Florida, Tampa, FL 33612, USA resolution morphological measures, like spectral-domain optical coherence tomography (SD-OCT), is an important step in improving our understanding of the correlation between different retinal parameter changes in DR.

We thought that it would be important to comment on some of the aspects of this work for the broader readership audience of the journal. Thus, the author's conclusion that "both fullfield ERG and central retinal thickness measurements have a limited clinical value in the staging of retinopathy in unselected diabetes patients" needs to be qualitied and placed in perspective. Thus, in terms of ERG responses, this assertion applies only to the ones analyzed in this study: photopic $30 \mathrm{~Hz}$ flicker and single-flash cone response. However, the limited clinical value of these two ERG responses can be debated further and some uncertainty about their lack of usefulness still remain. As the authors point out, when removing outliers, the association between $30 \mathrm{~Hz}$ flicker peak time and the degree of retinopathy becomes significant. Additionally, there are several limitations related to the patient population. First, all patients were type I diabetes and thus, may have been under better glucose blood monitoring control and clinical monitoring. Having said that, the level of HbAlc at the time of ERG testing was not known and this may have contributed to some of the variability in the responses, including the presence of outliers. Second, despite the relatively large total number of patients for this type of study $(n=151)$, in some of the categories (e.g., patients with DR level 4 - severe nonproliferative DR), a relatively small number of seven patients were recruited, or only $12.7 \%$ compared to the number of patients recruited in another category: patients with DR level 1 - no retinopathy $(n=55)$. Such an unbalanced patient distribution may have resulted in a higher threshold for significance, especially taking into account the necessity for age effects correction. This may partially explain why the present study did not find an association between photopic $30 \mathrm{~Hz}$ flicker peak time and DR, while two other similarly sized studies, Bresnick \& Palta 1987 [4] $(n=66)$ and Kim et al. 
1998 [5] ( $\mathrm{n}=178)$, did find an association. Thus, it is likely that an even larger and better balanced study in terms of DR severity would provide a more definitive answer about the correlation of standard photopic full-field ERG responses with DR severity.

Additionally, based on previously conducted ERG studies [2] and on some more recent experimental and clinical studies, it appears that the dark-adapted DR retina shows more functional pathologic changes compared to the light-adapted DR retina $[6,7]$. Thus, although the authors should be commended for trying to establish a shortened and easier-to-implement fullfield ERG protocol by using only the light-adapted ERG, this may turn out to be too difficult and such an approach may be viable only in conjunction with dark-adapted ERG.

Finally, it would be useful for the broader clinical audience to explain why the $30 \mathrm{~Hz}$ flicker response appears to be more sensitive to DR changes compared to single-flash cone response in this study and in other similar works. It can be hypothesized that the $30 \mathrm{~Hz}$ flicker response drives the retina to operate near the limit of its temporal resolution ability where any defects in the metabolic state of the tissue would be more pronounced and any deficit would be much easier to demonstrate. Direct support for this hypothesis comes from a study by Lobefallo et al. [8]. They studied flicker fusion frequency in children with diabetes mellitus without fluorescein angiography signs of DR and found that the flicker fusion frequency threshold dropped from a mean value of $38.7 \mathrm{~Hz}$ in control subjects to $33.9 \mathrm{~Hz}$ in patients with good metabolic control, and to $27.4 \mathrm{~Hz}$ in patients with poor metabolic control. These DR flicker fusion threshold values are very close to the $30 \mathrm{~Hz}$ ERG frequency used in this study and recommended by the International Society for Clinical Electrophysiology of Vision (ISCEV) standard [9] and this may explain the sensitivity of this ERG response in DR.

Another fascinating aspect of the study by Jansson et al. [3] is the significant association between the mean retinal thickness as measured by SD-OCT and the two photopic ERG responses: light-adapted 3 ERG (as defined by the ISCEV standard) and light-adapted $30 \mathrm{~Hz}$ flicker ERG response. In particular, the association between central retinal thickness and the light-adapted 3 ERG b-wave amplitude seems remarkable $(p=0.006)$ and carries a significant promise to be used as a biomarker or a secondary outcome measure in future clinical trials for treatment of DR. The full potential of the objective ophthalmic tests, such as full-field ERG or SDOCT in DR, can only be uncovered by conducting relatively large population-based clinical studies that include patients with different DR severity, and the current study by Jansson et al. [3] is an important step in that direction.

\section{References}

1. National Eye Institute (2015) Diabetic Retinopathy. https://www.nei. nih.gov/eyedata/diabetic\#2. Accessed 18 Apr 2015

2. Tzekov R, Arden GB (1999) The electroretinogram in diabetic retinopathy. Surv Ophthalmol 44:53-60

3. Jansson RW, Raeder MB, Krohn J (2015) Photopic full-field electroretinography and optical coherence tomography in type 1 diabetic retinopathy. doi:10.1007/s00417-015-3034-y

4. Bresnick GH, Palta M (1987) Temporal aspects of the electroretinogram in diabetic retinopathy. Arch Ophthalmol 105:660-664

5. Kim SH, Lee SH, Bae JY, Cho JH, Kang YS (1997) Electroretinographic evaluation in adult diabetics. Doc Ophthalmol 94:201-213

6. Arden GB, Sivaprasad S (2012) The pathogenesis of early retinal changes of diabetic retinopathy. Doc Ophthalmol 124:15-26

7. Heckenlively JR (2011) New concept: treating nonproliferative diabetic retinopathy with light adaptation of rods during sleep. Eye (Lond) 25:1533-1534

8. Lobefalo L, Verrotti A, Mastropasqua L, Chiarelli F, Morgese G, Gallenga PE (1997) Flicker perimetry in diabetic children without retinopathy. Can J Ophthalmol 32:324-328

9. McCulloch DL, Marmor MF, Brigell MG, Hamilton R, Holder GE, Tzekov R, Bach M (2015) ISCEV Standard for full-field clinical electroretinography (2015 update). Doc Ophthalmol 130:1-12 